\title{
Entretien avec Pierre Chauvin
}

\section{Pierre Chauvin}

\section{(2) OpenEdition \\ Journals}

Édition électronique

URL : http://journals.openedition.org/rfst/431

DOI : $10.4000 /$ rfst. 431

ISSN : 2492-3672

Éditeur

Espaces et SOciétés (UMR 6590)

\section{Référence électronique}

Pierre Chauvin, «Entretien avec Pierre Chauvin », Revue francophone sur la santé et les territoires [En

ligne], Genre, territoire et santé, mis en ligne le 21 décembre 2015, consulté le 06 avril 2021. URL: http://journals.openedition.org/rfst/431 ; DOI : https://doi.org/10.4000/rfst.431

Ce document a été généré automatiquement le 6 avril 2021.

\section{(c) (i) (2) (2)}

La Revue francophone sur la santé et les territoires est mise à disposition selon les termes de la Licence Creative Commons Attribution - Pas d'Utilisation Commerciale - Partage dans les Mêmes Conditions 4.0 International. 


\title{
Entretien avec Pierre Chauvin
}

\author{
Pierre Chauvin
}

\section{NOTE DE L'ÉDITEUR}

Propos recueillis par Sandrine Halfen, Audrey Bochaton, Clélia Gasquet-Blanchard

\section{Biographie}

1 Pierre Chauvin est Docteur en médecine et Docteur en épidémiologie et santé publique. Il est Directeur de recherche à l'Inserm et dirige actuellement l'Équipe de recherche en épidémiologie sociale (ERES) de l'Institut Pierre Louis d'Epidémiologie et de Santé Publique (UMRS 1136, Inserm \& Sorbonne Universités UPMC), à Paris.

2 Depuis 1997, ses travaux concernent les inégalités sociales de santé et les déterminants sociaux et territoriaux de la santé et du recours aux soins curatifs et préventifs en milieu urbain, notamment dans l'agglomération parisienne, avec un souci particulier porté aux populations vulnérables et aux processus de ségrégation socio-spatiale. Il codirige la cohorte Santé, inégalités et ruptures sociales (SIRS) depuis 2005.

\section{Le genre comme catégorie d'analyse en rapport avec la santé}

\section{En quoi le genre peut-il constituer une approche pertinente d'analyse des comportements de santé ?}

3 Les «comportements de santé » comme vous dites renvoient à plusieurs types de comportements. Dans le langage médical ou de santé publique, ils concernent les comportements dits "à risque ": les intoxications et les addictions (tabac, alcool, drogues...), les comportements alimentaires non (mal) sains, la sédentarité, etc. Dans le champ de la santé au travail, les comportements renvoient aux risques psychosociaux 
ou aux comportements et postures (contraintes) susceptibles de provoquer des troubles organiques. En santé mentale, certains comportements ou attitudes sont accessibles aux thérapies " comportementales" (justement). Tous ceux qui travaillent sur l'accès aux soins savent bien également qu'il s'agit d'un comportement et que ses déterminants ne sont pas qu'une question d'accessibilité financière ou géographique. Nos études, parmi d'autres, ont montré que "toutes choses égales par ailleurs" concernant la couverture maladie et les revenus des personnes, leurs recours aux soins dépendaient de leurs insertions sociales, de leur capital social, de leurs expériences passées de la maladie... des facteurs par ailleurs bien différents chez les hommes et les femmes. Chez les patients pris en charge, enfin, l'observance et l'adhésion à une démarche thérapeutique ou préventive est également un comportement de santé particulièrement important pour de nombreuses maladies chroniques ou accessibles à la prévention. Ce qui relie ces comportements, c'est que ce sont des comportements humains -pardon du truisme -et sont façonnés et influencés par un très grand nombre de déterminants, au rang desquelles des normes (collectives et individuelles) et des interactions sociales qui, tous ou la plupart, sont façonnés à un degré divers par des rapports de genre.

\section{Quelles sont les positionnements scientifiques actuels sur le genre en épidémiologie, notamment en épidémiologie sociale, voire en médecine sociale/santé publique?}

4 Le genre reste largement sous étudié. Même s'il est tout à fait admis en épidémiologie sociale qu'il est un déterminant social de la santé et qu'on observe effectivement, en France comme ailleurs, des particularités liées au genre (plus grand souci de soi, rapport au corps, à la maladie et à la médecine différents, recours aux soins plus élevé chez les femmes en sont autant d'exemples), cette question du genre reste peu abordée, peu systématiquement traitée et approfondie en épidémiologie sociale et en santé publique en France. Je vois plusieurs raisons à cela. D'une part, d'une façon générale, l'épidémiologie reste en France prisonnière d'un paradigme biomédical et - même si les choses ont beaucoup changé en 20 ans - l'importance et le poids des facteurs sociaux dans la santé restent souvent perçus comme moins déterminants, minoritaires et/ou moins accessibles au changement que les causes biologiques, perçues comme plus directes, plus déterministes, plus accessibles à l'intervention, au "progrès médical » (et, par là, plus sources d'innovation technologique et de profits). Pourtant, il a été montré que les déterminants sociaux et biologiques sont de "poids» en réalité identique : quand elle a été estimée, pour certaines maladies comme le cancer, la part «attribuable » à ces deux catégories de facteurs est du même ordre de grandeur. Audelà de cet exercice un peu artificiel, on sait bien également en santé publique qu'il est illusoire de distinguer les bénéfices attendus du " progrès médical » des enjeux sociaux de la santé : les uns sont modulés par les autres et inversement. D'autre part, il est certain que l'étude épidémiologique, donc statistique, des déterminants sociaux est complexe d'un point de vue méthodologique et coûteuse du point de vue des ressources à mobiliser en temps, en moyens financiers et humains, sans aucune mesure avec les études qualitatives. Elle nécessite des indicateurs et des outils quantitatifs pertinents, des données nombreuses, des méthodes statistiques parfois assez pointues et une "juste distance» entre réductionnisme (inhérent à l'approche statistique) et un holisme ontologique qui serait trop éloigné des exigences de l'épidémiologie (en termes 
de validité et de reproductibilité notamment). Enfin, les échanges disciplinaires entre les sociologues du genre et les épidémiologistes restent trop cantonnés dans le champ de la santé gynécologique, reproductive ou sexuelle; ça n'incite pas à développer des approches systématiques dans le champ de la santé en général, dans tous les autres champs - notamment des comportements de santé dont nous parlions.

\section{Quelle acception avez-vous du genre : en faites-vous un champ d'étude à part entière ou bien un objet présent dans toutes vos recherches?}

5 C'est à la fois un objet présent dans toutes nos recherches sans être un champ d'étude à part entière! Classiquement, on stratifie volontiers et assez systématiquement nos analyses selon le sexe des personnes et on observe parfois des interactions entre les facteurs sociaux que nous étudions et le sexe qui démontre statistiquement que les liens observés n'ont pas la même intensité ou ne sont pas de même nature dans les deux sexes. Ce sont des choses qu'on a bien étudié pour la dépression par exemple, ou les déterminants sociaux individuels et résidentiels de recours à certains soins de prévention ou de dépistage. En revanche, pour dire la vérité, nous restons très en-deçà d'une véritable analyse dans une perspective de genre, dans le sens où nous ne mettons guère les différences observées par sexe en lien avec les relations de pouvoirs ou les processus de discrimination ou de domination des femmes. Plusieurs explications à cela : nous ne sommes pas très à l'aise pour tester de telles hypothèses empiriquement et de façon inductive, en inférant statistiquement à partir d'observations nombreuses, ce qui est le raisonnement habituel de l'épidémiologie; il nous manque des données pour tester directement ces hypothèses dans ces termes de pouvoir et de discriminations (ou même de normes sanitaires et sociales en fonction du sexe); il nous manque aussi des alter égo en sciences sociales qui s'intéresse à ces questions dans une perspective partagée, à la fois populationnelle et appliquée. Il n'est pas si facile de mobiliser des chercheurs du genre vers des problématiques de santé publique, des travaux quantitatifs et des recommandations opérationnelles pour les politiques et les professionnels de santé.

\section{Comment l'épidémiologiste qui souhaite aujourd'hui intégrer les questions de genre doit-il s'y prendre?}

6 Bien entendu d'abord en développant des approches pluridisciplinaires, en se confrontant aux autres disciplines, en rapportant dans le champ de l'épidémiologie des constats des sciences sociales et en tentant, ce qui n'est pas simple, de les traduire en concepts mobilisables dans une analyse quantitative et en résultats utiles pour les pratiques de soins ou les politiques de santé publique. En gros, faire pour la problématique du genre ce qui a été fait pour et avec les problématiques sociologiques de la précarité ou des liens sociaux, par exemple. Une bonne porte d'entrée, en épidémiologie sociale, me semble être de comparer plus systématiquement, entre les hommes et les femmes, pourquoi l'amplitude des inégalités sociales propres à chaque sexe ne sont pas les mêmes entre les deux sexes, pourquoi ce ne sont pas les mêmes déterminants sociaux qui les façonnent et est-ce que les processus de discrimination ou de domination dont nous parlions peuvent (ou non) expliquer ces différences. L'intérêt 
de la démarche épidémiologique, issue du raisonnement statistique, est - me semble-til - de poser systématiquement une hypothèse et son hypothèse alternative, et de raisonner en termes de risques, éventuellement cumulatifs, compétitifs ou synergiques (interactions). Pour faire court, on ne cherchera pas à démontrer que des relations de genre façonnent les différences observées entre hommes et femmes (ce que les sciences humaines et sociales font très bien, et les approches qualitatives de façon très fine) mais plutôt si elles, plutôt que d'autres (biologiques notamment) le font et dans quelles proportions et avec quelle intensité en fonction de différentes caractéristiques (sociales, sanitaires ou biologiques) des femmes.

\section{Articuler genre, territoire et santé}

\section{Existe-t-il des spécificités féminines et masculines en termes de pratiques territoriales ? Comment ces différences peuvent-elles se traduire dans les comportements et les états de santé ?}

7 Les «pratiques territoriales » sont en effet, probablement, "genrées »; qu'il s'agisse des rapports au quartier de résidence - via, notamment, les rôles sociaux et les taches quotidiennes qui restent largement dévolues aux femmes: approvisionnement alimentaire, accompagnement scolaire des enfants, contacts de voisinage (autant de sources de "liens sociaux faibles ») - ou de la mobilité quotidienne, liée notamment aux trajets domicile-travail, dont on sait qu'il sont inégalement répartis en fonction du statut socio-économique, du niveau de qualification et des secteurs d'activité et donc, au moins par là, fonction du genre. Dès lors, les femmes (et notamment les moins mobiles d'entre elles, dont on a montré dans nos travaux que c'était volontiers les plus modestes et les plus pauvres) pourraient être plus sensibles que les hommes aux effets contextuels des caractéristiques de leur environnement de résidence. Parmi les caractéristiques des environnements de résidence, les expositions physiques sont de plus en plus étudiées mais, à ma connaissance, il n'y a pas encore eu d'études statistiques françaises qui croisent le concept de «justice environnementale » et la question du genre, notamment en ce qui concerne, en épidémiologie environnementale, ces expositions physiques (le bruit, la pollution, etc.). On sait enfin que les pratiques de et dans l'espace public sont également différentes chez les hommes et les femmes; celles-ci adaptant leurs comportements à certaines situations (par exemple d'insécurité). Il manque là aussi de travaux français sur les conséquences de ces appropriations et comportements différenciés dans l'espace public sur les comportements en lien avec la santé chez les femmes (activité physique, accès et utilisation des services notamment). Enfin, à la croisée des pratiques territoriales et des pratiques sociales, si on fait l'hypothèse que les informations et les normes de santé se construisent à la faveur des interactions sociales, je pense qu'il serait intéressant de mesurer les différences entre (certains) hommes et (certaines) femmes en matière de contacts sociaux quotidiens, notamment au travail, avec des personnes d'autres origines sociales qu'eux/elles-mêmes. S'agissant des catégories populaires, on peut en effet penser que certains emplois féminins peu qualifiés se traduisent par moins (par exemple dans le secteur du nettoyage en horaires décalés) ou au contraire par plus (par exemple dans le secteur de l'aide aux personnes ou du commerce) de contacts diversifiés que des emplois masculins de même niveau de faible qualification. 
A votre avis, quel risque y a-t-il à spatialiser les questions de genre, particulièrement au regard des questions de santé qui peuvent dans certaines approches biomédicales participer à un déterminisme de genre?

8 Je n'en vois guère. Au contraire, il y a là des résultats possiblement intéressants en termes d'aménagement urbain de l'espace public, d'aménagement du territoire en termes d'offres d'équipement et de services de proximité (y compris des services de santé) et, d'une façon générale, de compréhension du rôle des pratiques spatiales différenciées dans les inégalités sociales de genre en santé.

Vous travaillez à l'articulation entre les notions de territoire et santé. A votre avis, en quoi l'approche de genre peut-elle aider à comprendre les liens entre le territoire et la santé ?

9 De telles approches peuvent non seulement aider à comprendre mais, en amont, à révéler (à proprement parler) des inégalités socioterritoriales de santé qui ne s'observeraient pas si on ne fait pas attention à analyser systématiquement les différences hommes/femmes et à rechercher systématiquement des interactions en fonction du sexe.

\section{L'observation d'inégalités de santé entre les hommes et les femmes relève-t-elle, outre de prédispositions biologiques, de constructions sociales et territoriales de genre?}

10 Ça me semble absolument évident, qu'il s'agisse des influences «culturelles » (au sens large), normatives, familiales (au sens de l'acquisition dans l'enfance et l'adolescence de normes de santé et de recours aux soins et/ou à l'âge adulte au sens de la distribution des rôles sociaux intrafamiliaux), ou encore des rapports au corps, à la souffrance, à la résistance physique, mais aussi des expériences vécues (pour soi ou des proches) de la maladie et de rapport aux soins de santé, des capacités propres (et/ou admises par la société) aux nécessaires reconstructions identitaires qu'impliquent la maladie, etc. De l'adhésion aux messages de prévention primaire aux recours aux soins curatifs, du souci de soi aux capacités de rétablissement après la guérison, tous ces déterminants psychosociaux relèvent de constructions sociales de genre. L'idée n'est même pas de les opposer aux prédispositions biologiques (et de répéter ici l'opposition stérile entre nature et culture) mais de comprendre qu'elles sont absolument liées. Les premières remodèlent les secondes (elles peuvent les potentialiser ou au contraire faire écran) et les secondes modulent aussi très probablement les capacités biologiques.

\section{Quels sont les principaux enseignements sur le genre de la cohorte SIRS relatifs aux liens entre territoire et santé ?}

11 La cohorte SIRS (Santé, inégalités et ruptures sociales) suit depuis 2005 un échantillon représentatif de la population de 50 quartiers du Grand Paris $(75,92,93,94)$, soit 
3000 ménages, dans le cadre d'un programme de recherche pluridisciplinaire associant des épidémiologistes de mon équipe de recherche à l'INSERM, des sociologues (notamment Serge Paugam et Isabelle Parizot au Centre Maurice Halbwachs du CNRS) et des géographes (notamment Julie Vallée de l'Unité Géographie Cités du CNRS). Il s'agit de la première cohorte représentative de la population générale constituée spécifiquement pour l'étude des déterminants sociaux et territoriaux de la santé et du recours aux soins. De nombreuses caractéristiques sociales et sanitaires sont recueillies auprès des personnes enquêtées. Trois dimensions sont particulièrement étudiées et ont justifié la mise en place de cette cohorte: les effets des liens sociaux et de l'insertion dans les différentes sphères de sociabilité sur les comportements liés à la santé (incluant les recours aux soins curatifs et préventifs, en particulier les dépistages du VIH et des cancers gynécologiques), la santé des immigrés et des Français issus de l'immigration et, enfin, le rôle des espaces de vie des populations (appréhendés à l'aide d'un système d'information géographique dans lequel l'adresse de résidence des personnes interrogées ainsi que celle de certaines de leurs destinations quotidiennes sont intégrées). C'est à partir de ces données que nous avons observé les différences de mobilité quotidienne en fonction du sexe et du statut socio-économique que je mentionnais à l'instant. On y observe également de fortes différences de prévalence entre hommes et femmes en matière de santé ressentie, de santé mentale et de comportements de recours aux soins. Chez les femmes plus spécifiquement, nous avons été sans doute les premiers à décrire un gradient social qui distingue les femmes françaises de la population majoritaire (selon la terminologie de l'INED, c'est-à-dire françaises ni immigrées ni descendantes d'immigrés), les femmes françaises nées de parents immigrées et les immigrées. Pour de nombreux états de santé et recours aux soins, les immigrées sont, globalement, dans la situation la plus péjorative et les Françaises issues de l'immigration restent dans une situation moins favorable que la population majoritaire. Ces différences s'expliquent en grande partie, mais pas complètement, par des différences de statut socioéconomique; la part non expliquée renvoie probablement à des facteurs psychosociaux et culturels qu'il serait intéressant d'investiguer de manière plus approfondie. Un autre exemple tiré de SIRS concerne l'insécurité alimentaire (la situation dans laquelle la possibilité de s'approvisionner en nourriture suffisante et adéquate d'un point de vue nutritionnel et de façon socialement acceptable - c'est-à-dire sans le recours à la mendicité, au vol, aux dons ou aux aides alimentaires - est limitée ou incertaine). Les travaux de Judith MartinFernandez dans mon équipe ont montré que sa prévalence dans Paris et sa proche couronne (globalement estimée à $8 \%$ des ménages, $14 \%$ des ménages vivant en Zone urbaine sensible, près de $25 \%$ des ménages immigrés maghrébins ou africains, et $27 \%$ des ménages bénéficiant de minima sociaux) était plus élevée quand le chef de famille était une femme et, notamment, dans les ménages monoparentaux. Chez les femmes, et chez elles seulement, on observait également, une association entre insécurité alimentaire sévère (c'est-à-dire avec restrictions alimentaires et réduction des apports) et obésité. Une des explications est sans doute liée au fait que les femmes sont, dans le ménage, les premières à réduire leurs apports et changer leur alimentation quand l'argent manque (vers une alimentation plus densément énergétique et/ou de moins bonne qualité, avec éventuellement des alternances de " gavage » et de restriction), en préservant le plus possible celle des autres membres du ménage (les hommes et, surtout, les enfants). Les inégalités territoriales d'insécurité alimentaire étaient très majoritairement expliquées par des effets de composition (c'est-à-dire liées aux 
caractéristiques socioéconomiques des habitants). Pour autant, une fois cet effet pris en compte dans les modèles statistiques, nous avons montré qu'habiter dans un quartier très doté en supérettes (il s'agit plutôt de quartiers dans Paris intra-muros) ou dans un quartier peu dotés en commerce de bouche de détails (plutôt dans certains quartiers de banlieue) étaient des facteurs de risque associés à l'insécurité alimentaire. La première situation pourrait s'expliquer par les coûts pratiqués dans ces superettes (plus élevé que dans les super et hypermarchés); la seconde situation renverrait plutôt aux situations de "déserts alimentaires " où l'absence de commerces de bouche de détail réduit l'accès à une offre alimentaire diversifiée et peu coûteuse (en l'absence de moyens de transport par exemple). Comme on sait que les femmes pauvres de ces quartiers périphériques sont les moins mobiles et ont le moins de moyens de locomotion, on peut faire l'hypothèse que ces situations de "déserts alimentaires " impactent particulièrement ces femmes et particulièrement les familles monoparentales pauvres.

\section{RÉSUMÉS}

Cet entretien a été réalisé avec Pierre Chauvin, directeur de recherches à l'Inserm, et directeur de l'équipe de recherche en épidémiologie sociale (ERES) de l'Institut Pierre Louis d'Épidémiologie et de Santé Publique (UMRS 1136, Inserm \& Sorbonne Universités UPMC), à Paris. Pierre Chauvin conduit depuis près de vingt ans des travaux sur les inégalités sociales de santé et les déterminants sociaux et territoriaux de la santé et du recours aux soins. Il co-dirige la cohorte Santé, inégalités et ruptures sociales (SIRS) depuis 2005.

Selon lui, les comportements de santé, au sens large, sont façonnés et influencés par un très grand nombre de déterminants, au rang desquelles des normes (collectives et individuelles) et des interactions sociales qui, tous ou la plupart, sont façonnés à un degré divers par des rapports de genre. Le genre reste largement sous-étudié en épidémiologie sociale. Même s'il est tout à fait admis qu'il est un déterminant social de la santé et qu'on observe effectivement, en France comme ailleurs, des particularités liées au genre, cette question du genre reste peu abordée en épidémiologie sociale et en santé publique en France. Une meilleure prise en compte de celui-ci en épidémiologie nécessiterait de développer des approches pluridisciplinaires, en se confrontant aux autres disciplines, en rapportant dans le champ de l'épidémiologie des constats des sciences sociales et en tentant, ce qui n'est pas simple, de les traduire en concepts mobilisables dans une analyse quantitative et en résultats utiles pour les pratiques de soins ou les politiques de santé publique.

This interview was conducted with Pierre Chauvin, MD, PhD, Director of research at the French Institute for Health and Medical Researches (Inserm), and Director of the Social Epidemiology Research Center (ERES) of Pierre Louis Institute of Epidemiology and Public Health (UMRS 1136, Inserm \& UPMC Sorbonne University) in Paris.

Pierre Chauvin conducted research for nearly twenty years on health inequalities and social and territorial determinants of health and access to health care. Since 2005, he is the co-founder and main investigator of the SIRS cohort (which means Health, Inequalities and Social Disruptions) conducted in Paris region among 3,000 inhabitants within 50 neighborhoods. 
According to Chauvin, health behaviors, in the broad sense, are shaped and influenced by a large number of determinants, among which standards (collective and individual) and social interactions, that are all, or almost all, shaped to varying degrees by gender relations. Nevertheless, the gender issue remains largely understudied in social epidemiology and public health in France, although it is well recognized that it is a social determinant of health and that distinctive features related to gender are documented in France as elsewhere. To better take gender into account in epidemiology, it would require the development of multidisciplinary approaches, and to review findings from social sciences in the field of epidemiology and to try to translate these findings into concepts that could be use in quantitative analyses and in useful results for public health policy.

The SIRS cohort was created especially to study the role of the living environment in individuals' health and behaviors related to health (prevention, health care, etc.). From SIRS data, differences in daily mobility were observed by gender and socioeconomic status, probably emphasizing gendered territorial practices. Differences can be explained by the relationships with the neighborhood, especially with the gendered social roles and behaviors and the division of everyday tasks which remain largely assigned to women, or daily mobility, particularly related to commuting, which is known is unevenly distributed by socio-economic status, level of qualification and sectors and, thus, are largely dependent on gender. Therefore, women may be more sensitive than men to the effects of contextual characteristics of their environment on health, especially for the least mobile of them, which has been shown with SIRS data that it was usually the poorest ones.

\section{AUTEUR}

\section{PIERRE CHAUVIN}

Directeur de recherches à l'Inserm, et directeur de l'équipe de recherche en épidémiologie sociale (ERES) de l'Institut Pierre Louis d'Épidémiologie et de Santé Publique (UMRS 1136, Inserm \& Sorbonne Universités UPMC), à Paris 